\title{
Gestaltic Grouping of Line Segments
}

\author{
Boshra Rajaei ${ }^{1,2}$, Rafael Grompone von Gioi ${ }^{2}$ \\ ${ }^{1}$ Sadjad University of Technology, Mashhad, Iran (b.rajaei@sadjad.ac.ir) \\ ${ }^{2}$ CMLA, ENS Cachan, France (grompone@cmla.ens-cachan.fr) \\ Communicated by Pablo Musé Demo edited by Boshra Rajaei
}

\begin{abstract}
Using simple grouping rules in Gestalt theory, one may detect higher level features (geometric structures) in an image from elementary features. By recursive grouping of already detected geometric structures a bottom-up pyramid could be built that extracts increasingly complex geometric features from the input image. Taking advantage of the (recent) advances in reliable line segment detectors, in this paper, we propose three feature detectors along with their corresponding detailed algorithms that constitute one step up in this pyramid. For any digital image, our unsupervised algorithm computes three classic Gestalts from the set of predetected line segments: good continuations, non-local alignments, and bars. The methodology is based on a common stochastic a contrario model yielding three simple detection formulas, characterized by their number of false alarms. This detection algorithm is illustrated on several digital images.
\end{abstract}

\section{Source Code}

The Matlab source code implementing the proposed algorithms, which is part of this publication, is accessible at corresponding IPOL web page. ${ }^{1}$

Keywords: Gestalt theory; good continuation; alignment; parallelism; line segment; nonaccidentalness principle; a contrario detection; number of false alarms (NFA)

\section{Introduction}

A basic task of the visual system is to group fragments of a scene into objects and to separate one object from the others and from their background. Perceptual grouping has inspired many researches in psychology in the past century. At the beginning of the 20th century, a systematic approach for investigating human perception was introduced under the name of Gestalt theory [28, 18, 26, 27]. The Gestalt school proposed the existence of a short list of grouping laws governing visual perception. The Gestalt laws include, but are not limited to, similarity, proximity, connectedness and

\footnotetext{
${ }^{1}$ https://doi.org/10.5201/ipol.2018.194
} 
good continuation. Gestaltists argue that partial grouping laws recursively group image elements to form more organized Gestalts. But the grouping laws were qualitative and lacked a quantitative formalization.

Since its origins, computer vision has been interested in Gestalt laws and there have been several attempts to formalize aspects of the Gestalt program [17, 25]. We will concentrate on a particular approach $[8,9]$ which has led to the conception of several Gestalt detectors to organize meaningful geometric structures in a digital image. Based on the non-accidentalness principle [17, 23, 3], an observed structure is considered meaningful when the relation between its parts is too regular to be the result of an accidental arrangement of independent parts. This is the rationale behind the $a$ contrario model for determining potential Gestalts which is formulated in the next section.

The a contrario methodology has also been used in [16], inspired by the good continuation Gestalt principle, to propose an algorithm to detect alignments of points in a point pattern. The detected point alignments are further employed toward a nonparametric vanishing point estimating algorithm again based on the non-accidentalness principle [15]. Based on the same principle, an automatic line segment detector (LSD) is provided in $[11,12]$ with linear time complexity. The same approach is used in the EDLines straight line calculator [1] but with the difference that the latter algorithm starts from an edge drawing output while the former is based on image level lines. Similar ideas were also proposed to detect circles and ellipses [20,2]. Level lines are employed to detect good continuations and image corners in [6]. Using edge pieces (edgelets) the authors of [29] adopt a featured a contrario scheme to extract the strong edges and eliminate irrelevant and textural ones. Extracting an object of interest from textural background or from outlier points is studied in $[13,10]$ where, for instance, fixed contrast spots are detected in mammographic images.

Many image matching algorithms are also based on an a contrario model. In [21] given a set of query descriptors and candidate descriptors, a match between a query descriptor, $a$, and a candidate, $b$, is reported if it is unlikely that a generic random descriptor (that is defined using mutual independent random variables) is closer to $a$ than $b$. The $a$ contrario matching of blocks between two frames in a stereo image pair is studied in [24]. An agent-based hierarchical object matching using SIFT points is addressed in [4]. Finally, the a contrario method has been applied to the problem of shape element recognition using a database of desirable shapes [19].

The a contrario framework was used for image segmentation in [5]. To obtain robust segments, the authors suggest a combination of the a contrario approach and of Monte-Carlo simulation. Also, to solve the hierarchical segmentation issue, the authors of [7] suggest two a contrario criteria for measuring region and merging meaningfulness based on homogeneity and boundary contrast.

In this paper, we start from the "partial Gestalts" constituted by the line segments detected by LSD and explore if they can be used as basic elements for more elaborate Gestalt groups such as long straight lines, good continuations and parallel line segments. Toward this aim, an a contrario model is adopted on the line segment distribution. It is used repeatedly for detecting the mentioned structures as non-accidental. Figure 1 represents instances of the three Gestalts of interest in this paper. We shall observe in most images that, with the exception of a few isolated line segments, all line segments are classified precisely and hence the algorithm forms another level of segmentation pyramid toward a complete analysis and understanding of each image's structure. The main advantage of this approach is its low time complexity due to the exploitation of line segments as input structures which are far less numerous than the unstructured set of all image pixels. A preliminary version of this work was published in [22].

The rest of this paper is organized as follows. Section 2 introduces the theoretical formulation of the proposed method. Section 3 describes the resulting algorithm. Some results are shown and commented in Section 4 and Section 5 concludes the paper. 


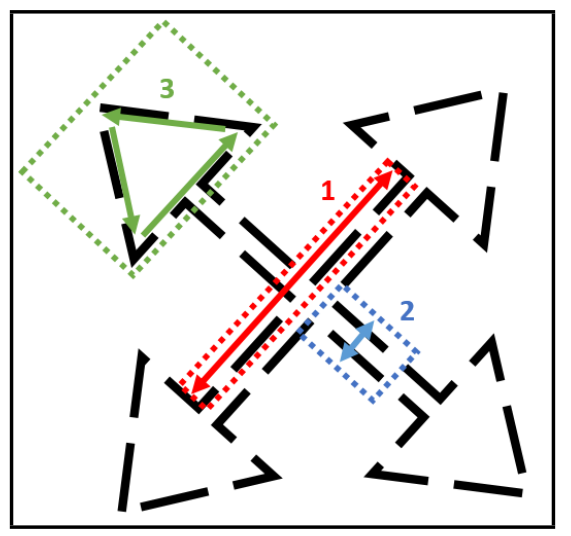

Figure 1: Instances of a non-local alignment (1), a bar (2) and a good continuation (3) Gestalts.

\section{$2 \quad$ A Contrario Model}

The a contrario detection approach has a probabilistic basis. Consider an event of interest $e$. According to the non-accidentalness principle, $e$ is meaningful if its expectation is low under a stochastic background (or a contrario) model $H_{0}$. The stochastic expectation of an event is called its number of false alarms (NFA) and is defined as

$$
\operatorname{NFA}(e)=N_{\text {test }} P_{H_{0}}(e)
$$

where $N_{\text {test }}$ indicates the number of possible occurrences of $e$ and $P_{H_{0}}(e)$ is the probability of $e$ happening under $H_{0}$. A relatively small NFA implies a rare event $e$ under the a contrario model and therefore, a meaningful one. An event $e$ is called $\epsilon$-meaningful if $\operatorname{NFA}(e)<\epsilon$.

In this paper, three types of line segment events are under focus: non-local alignments, good continuations and parallelism. Let us consider a set of $N$ oriented line segments $l_{1}, \ldots, l_{N}$ observed in a rectangular domain of size $n \times m$. The adopted a contrario model $H_{0}$ is a set of $N$ stochastic line segments, with independent and uniformly distributed tips on the image domain. Here, we provide a formal definition of each event.

Definition 1. A sequence of $k$ line segments $l_{a_{1}}, l_{a_{2}}, \ldots, l_{a_{k}}$ form a potential good continuation event $\wp^{k, \rho, \theta}$ if and only if $D\left(l_{a_{1}}, l_{a_{2}}, \ldots, l_{a_{k}}\right)<\rho$ and $\angle\left(l_{a_{1}}, l_{a_{2}}, \ldots, l_{a_{k}}\right)<\theta$ for predefined constant $\rho$ and $\theta$ thresholds.

In the above definition $D(\cdot)$ and $\angle(\cdot)$ denote respectively the maximum of the distances and the maximum of the angles between successive oriented line segments in a sequence. The two threshold values $\rho$ and $\theta$ limit the search space around each line tip for finding close line segments as represented in Figure 2.

A small fixed margin $\lambda(\lambda=5)$ is allowed to deal with possible misalignments in the LSD outputs. This special adopted sector is denoted by $\mathcal{S}$ in the sequel. Let us point out that the primary $l_{1}, \ldots, l_{N}$ are oriented and hence have ordered tips. This order is taken into account in $D(\cdot), \angle(\cdot)$ and similar later calculations.

Definition 2. A good continuation with $\theta=3^{\circ}$ is called a non-local alignment event $\zeta^{k, \rho}$.

Definition 3. Two line segments or non-local alignments $l_{i}$ and $l_{j}$ are said to form a bar $\Im^{\rho}$ if and only if $D_{m}\left(l_{i}, l_{j}\right)<\rho$ and $\pi-\Delta \theta \leq \angle\left(l_{i}, l_{j}\right) \leq \pi+\Delta \theta$ for $\Delta \theta=3^{\circ}$ and $\rho$ is a predefined constant threshold. 


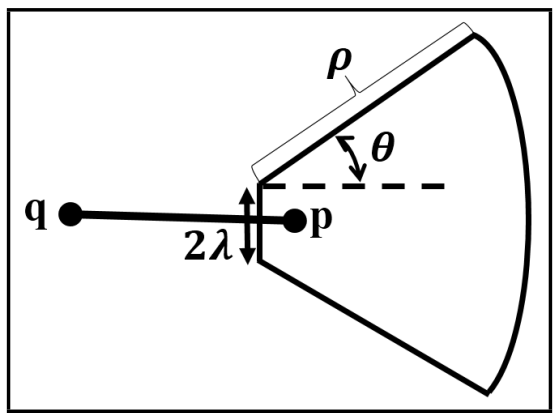

Figure 2: Given the endpoint $p$ of a line segment, this figure defines the domain $\mathcal{S}$ around $p$, in which the presence of successive line segment endpoints is searched by the algorithm. This search space depends on three parameters, $\rho, \theta$ and $\lambda$.

Here, $D_{m}(\cdot)$ stands for the mutual distance operator which is calculated as the maximum distance between the respective tips of the two line segments constructing the bar. The $\Delta \theta$ parameter is defined to cope with the sampling effect in digital images. Note that the orientation of line segments is considered in $D_{m}(\cdot)$ calculation. More precisely, the first tip of $l_{i}$ is paired with the second tip of $l_{j}$ and vice versa.

For the NFA calculation of a good continuation, according to (1), we have two parts: the number of tests and the probability of the geometric event under $H_{0}$. Here, $N_{\text {test }}$ is about all possible sequences of $k$ line segments out of overall $N$ line segments in the image which is $\frac{N !}{(N-k) !}$. We note $c_{1}$ the number of different pairs of $\rho$ and $\theta$ values potentially tried which is independent of $N$. The details of setting these two parameters are explained in Section 3. Therefore, $N_{\text {test }}=c_{1} \cdot \frac{N !}{(N-k) !}$.

For the probability term of $P_{H_{0}}\left(\wp^{k, \rho, \theta}\right)$, we assume in our a contrario model that all line segments tips are uniform i.i.d. spatial variables in the image domain and the line angles are uniformly distributed in the $[0,2 \pi]$ interval. Note that both of these assumptions are not simultaneously realizable because of boundary effects. Nevertheless for segments with limited lengths and large image domains, these assumptions are a valid approximation. Let us first compute the probability that two line segments be in good continuation in the sense specified in Figure 2 and with parameters $(\rho, \theta)$. We shall then extend the computation to $k$ line segments. When $\theta$ is expressed in radians, $\Pi_{s} \simeq \frac{\theta \rho^{2}}{m n}$ is the probability that the tip of the second line segment falls into $\mathcal{S}^{\rho, \theta}$ around the tip of the first one $^{2}$ and $\Pi_{a} \simeq \frac{\theta}{\pi}$ is the probability that a maximum angle of $\theta$ occurs between the two line segments. Therefore, $P_{H_{0}}\left(\wp^{2, \rho, \theta}\right)=\Pi_{s} \Pi_{a}^{3}$. Consequently, for $k-1$ junctions in a sequence of $k$ line segments we have $P_{H_{0}}\left(\wp^{k, \rho, \theta}\right)=\left(\Pi_{s} \Pi_{a}\right)^{k-1}$.

Finally, we may approximate the number of false alarms (NFA) associated to a good continuation event $\wp^{k, \rho, \theta}$ according to Definition 1 as

$$
\mathrm{NFA}^{G}(k, \rho, \theta)=c_{1} \cdot \frac{N !}{(N-k) !} \cdot\left(\frac{\theta \rho^{2}}{m n} \cdot \frac{\theta}{\pi}\right)^{k-1}
$$

Similarly, since non-local alignments, $\zeta^{k, \rho}$, are special types of good continuations, their NFA calculation is straightforward

$$
\mathrm{NFA}^{A}(k, \rho)=c_{2} \cdot \frac{N !}{(N-k) !}\left(\frac{2 \lambda \rho}{m n} \cdot \frac{\theta}{\pi}\right)^{k-1},
$$

\footnotetext{
${ }^{2}$ Since $\lambda$ is relatively small, we use this simple tight upper bound for $\Pi_{s}$. We also neglect the fact that the angular sector may sometimes fall outside the image domain, with area $m n$.

${ }^{3}$ Note that the angle of the line segments is determined by both tips, which are assumed independent in $H_{0}$. Therefore, to simplify the notation, except for the segments close to image boundaries, we can effectively suppose that the two conditions are independent.
} 
where constant $c_{2}$ is the number of different $\rho$ values we actually try. The only point is that for small $\theta=3^{\circ}, \lambda$ is not negligible anymore. Indeed, for small angles, $\mathcal{S}^{\rho, \theta}$ turns into a rectangle with dimensions $2 \lambda$ by $\rho$. Accordingly, we have $\Pi_{s}=\frac{2 \lambda \rho}{m n}$.

Eventually, the number of false alarms of parallel line (bar) event, $\Im^{\rho}$, according to Definition 3 is equal to

$$
\mathrm{NFA}^{B}(\rho)=c_{2} \cdot \frac{N(N-1)}{2}\left(\frac{\Delta \theta \rho^{2}}{m n}\right)^{2} .
$$

Using similar reasoning for the case of parallel lines, since we only have two line segments or non-local alignments, the $N_{\text {test }}$ term reduces to $\frac{N(N-1)}{2}$. If we consider angle and distance constraints over both corresponding tips, we have $\Pi_{a} \simeq\left(\frac{\Delta \theta}{\pi}\right)^{2}$ and $\Pi_{s} \simeq\left(\frac{\pi \rho^{2}}{m n}\right)^{2}$. Therefore, the overall NFA is formulated as (4).

\section{The Algorithm}

As mentioned earlier, by employing line segments as initial groups it is possible to detect more organized and sophisticated Gestalts. In this paper, we exploit the LSD algorithm [12] to produce $l_{1}, l_{2}, \ldots, l_{N}$ initial oriented line segments. The next step is finding all possible instances of our three Gestalts of interest in the form of sequences of line segments or chains.

Definition 4. A good continuation (Definition 1) chain $\wp^{k, \rho, \theta}$ is meaningful if and only if $N F A^{G}(k, \rho, \theta)<$ $\epsilon$ and there exists no subchain of $\wp$ with smaller NFA.

Meaningful non-local alignments and bars follow the same definition. We assume $\epsilon=1$ as a simple way to allow less than one false alarm on average for each Gestalt type per image [9].

Algorithm 1 describes the overall steps for calculating good continuation events. Using $\rho$ and $\theta$ thresholds for controlling the distance and angle between line segments, first, in Algorithm 2 an adjacency matrix, $\mathcal{A}$, is defined between input line segments, $\mathcal{L}$. Each entry $\mathcal{A}(i, j)$ represents the distance and angle between $i$ and $j$ line segments. In the second step, Algorithm 3 forms all the chains up to $K$ line segments. Here, parameter $K$ is defined in order to control the complexity of the algorithm. Furthermore, for each line tip we only consider up to three closer tips to make the algorithm even faster. Finally, meaningful good continuations are calculated according to Definition 4 in Algorithm 4.

In order to give preference to smoother continuations with closer tips, instead of only one value of $\rho$ and $\theta$ thresholds in the NFA calculation, we consider $n_{\rho}$ and $n_{\theta}$ predefined thresholds. Then, for each good continuation chain, and before calculating NFA, the smallest pair of $\left(\rho_{i}, \theta_{j}\right)$ that $D\left(l_{i}, l_{i+1}, \ldots, l_{j}\right)<\rho_{i}$ and $\angle\left(l_{i}, l_{i+1}, \ldots, l_{j}\right)<\theta_{j}$ is computed and employed in the NFA formula. According to this consideration, and also considering the maximum number of line segments in a chain, we have $c_{1}=K \times n_{\rho} \times n_{\theta}$ in (2) and (3).

By calling Algorithm 1 with $\theta=0.05$ (e.g. $3^{\circ}$ ) and replacing all $\mathrm{NFA}^{G}$ with $\mathrm{NFA}^{A}$, the same procedure yields non-local alignment gestalts. Non-local alignments and initial line segments are then fed to Algorithm 5 to calculate parallel line events. Due to digital sampling errors two line segments $l_{i}$ and $l_{j}$ are considered parallel if $\pi-\Delta \theta \leq \angle\left(l_{i}, l_{j}\right) \leq \pi+\Delta \theta$ where $\angle\left(l_{i}, l_{j}\right)$ is the angle between line segments. Here, $\Delta \theta$ is set to 0.05. The $\rho$ threshold in (4) is tested over $n_{\rho}$ different values similar to good continuations. Therefore, in parallel line NFA formula we have $c_{2}=n_{\rho}$.

Finally, by merging all meaningful Gestalts we obtain a drawing of the input image. The next section addresses the efficiency of the proposed approach applied on real-world images. 

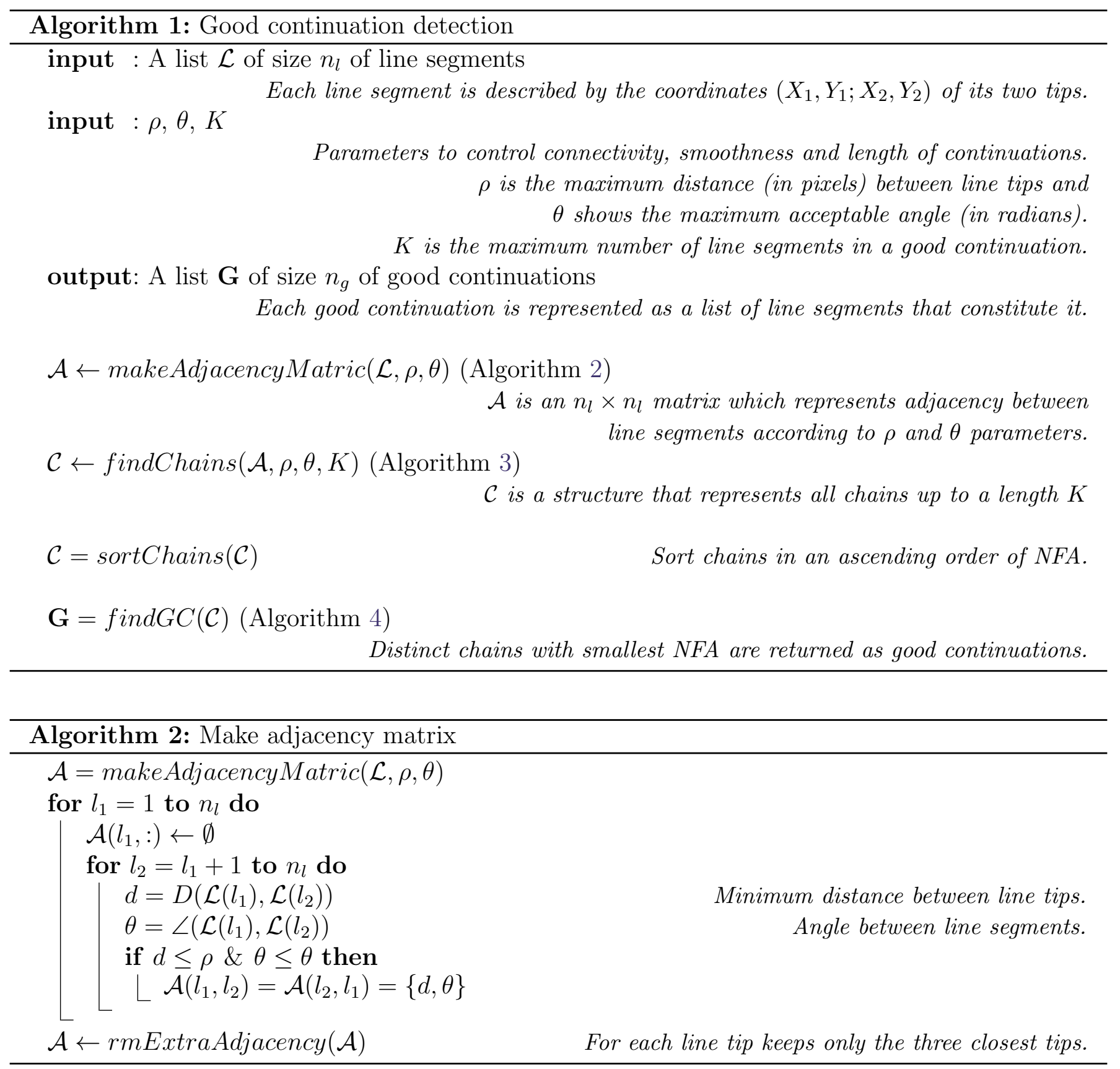

\section{Experimental Results}

Man-made structures like buildings, furniture and natural scenes present many good continuations in the form of line segments and curves or basic geometrical shapes like rectangles and parallelograms. To organize these structures using the proposed algorithm in this paper, we first applied LSD [12]. Afterwards, the two initial thresholds $\theta$ and $\rho$ in Definitions 1 to 3 must be determined. These two parameters limit the number of chains that later are considered by the a contrario model. The parameter $\theta$ controls the smoothness of output Gestalts while $\rho$ is a parameter proportional to the image size restricting the maximum acceptable distance between line segments of a candidate chain. In the sequel and to detect all kinds of parallelogram shapes, $\theta$ is set to $150^{\circ}$ and $\rho$ is experimentally fixed at $\min (10,\lceil 0.1 \times \max (m, n)\rceil)$ pixels by default. In Algorithm 1 , the maximum number of line segments in a good continuation, $K$, is set to 10 . Additionally, the angle threshold between line segments of a bar in Algorithm 5 is set to $5^{\circ}$. 

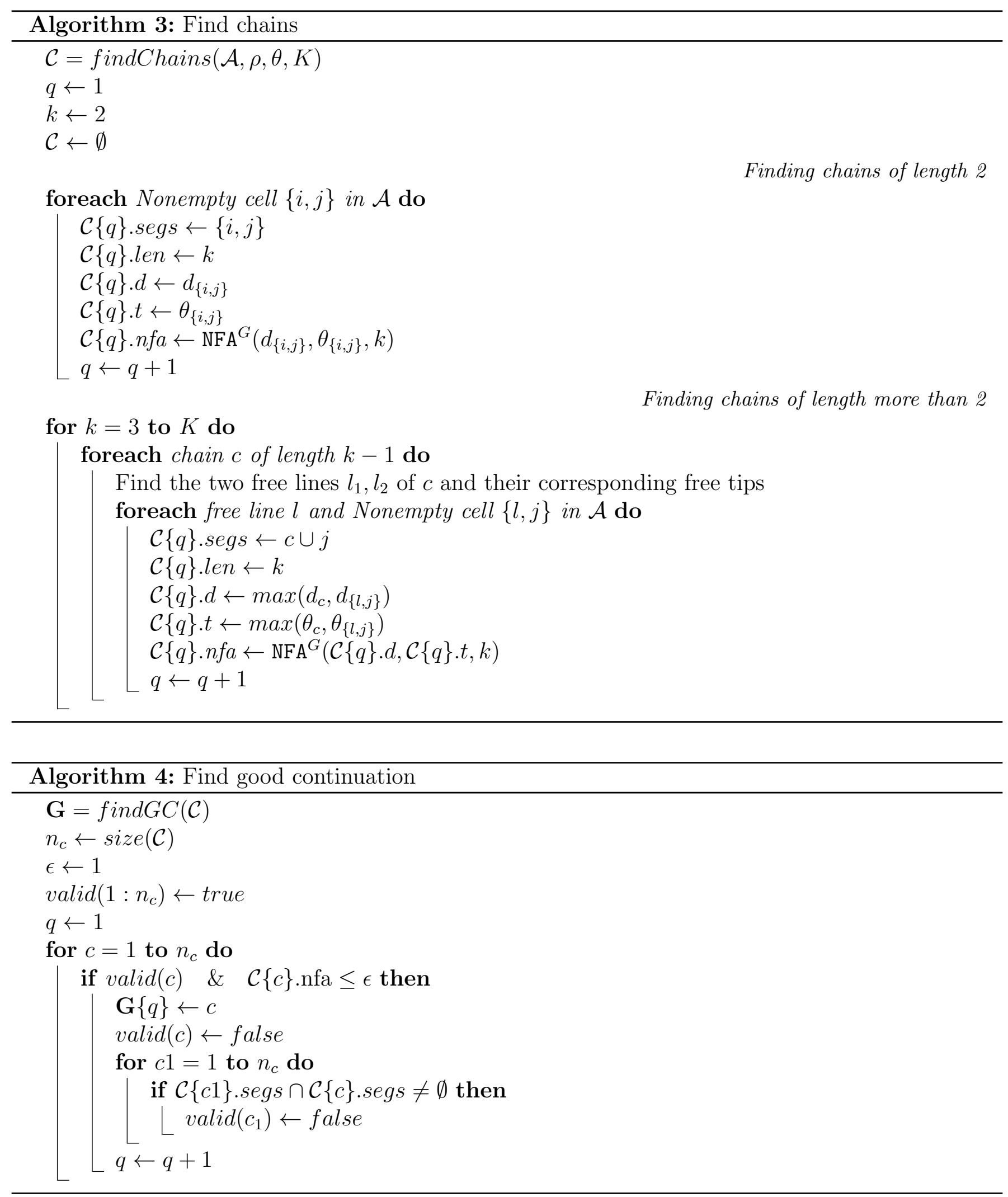

Figure 3 shows the result of the joint application of all feature detectors on an image. In each output image, organized Gestalts are depicted with different colors and finally the residual line segments are shown in the last image. Note that the detected structures could be grouped again using similar methods into more complex structures. The line segments that remained ungrouped consist mostly of isolated line segments that are not expected to belong to any organized structure. 


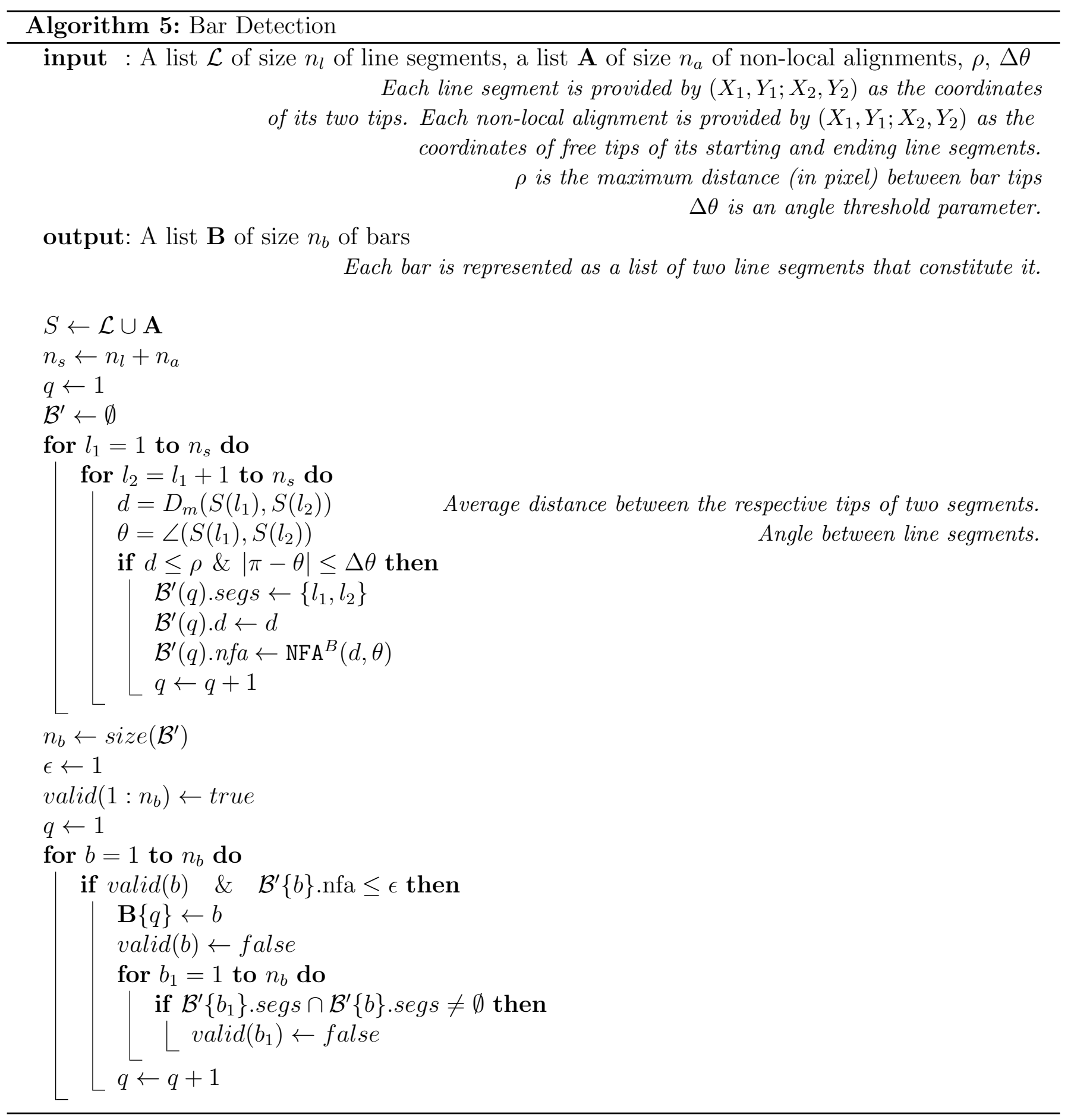

More results on more real-world or synthetic images are provided in Figure 4 and Figure 5.

\section{Conclusion}

The three detectors proposed in this paper represent one step up in the Gestalt grouping pyramid. The good experimental point is that few line segments are generally left out unorganized, a requirement that was called "articulation without rest" in the Gestalt literature [14]. Clearly this step up must be completed by further bottom up grouping. For example, good continuation curves present gaps that must be completed with irregular contours. Other gaps in good continuations or align- 


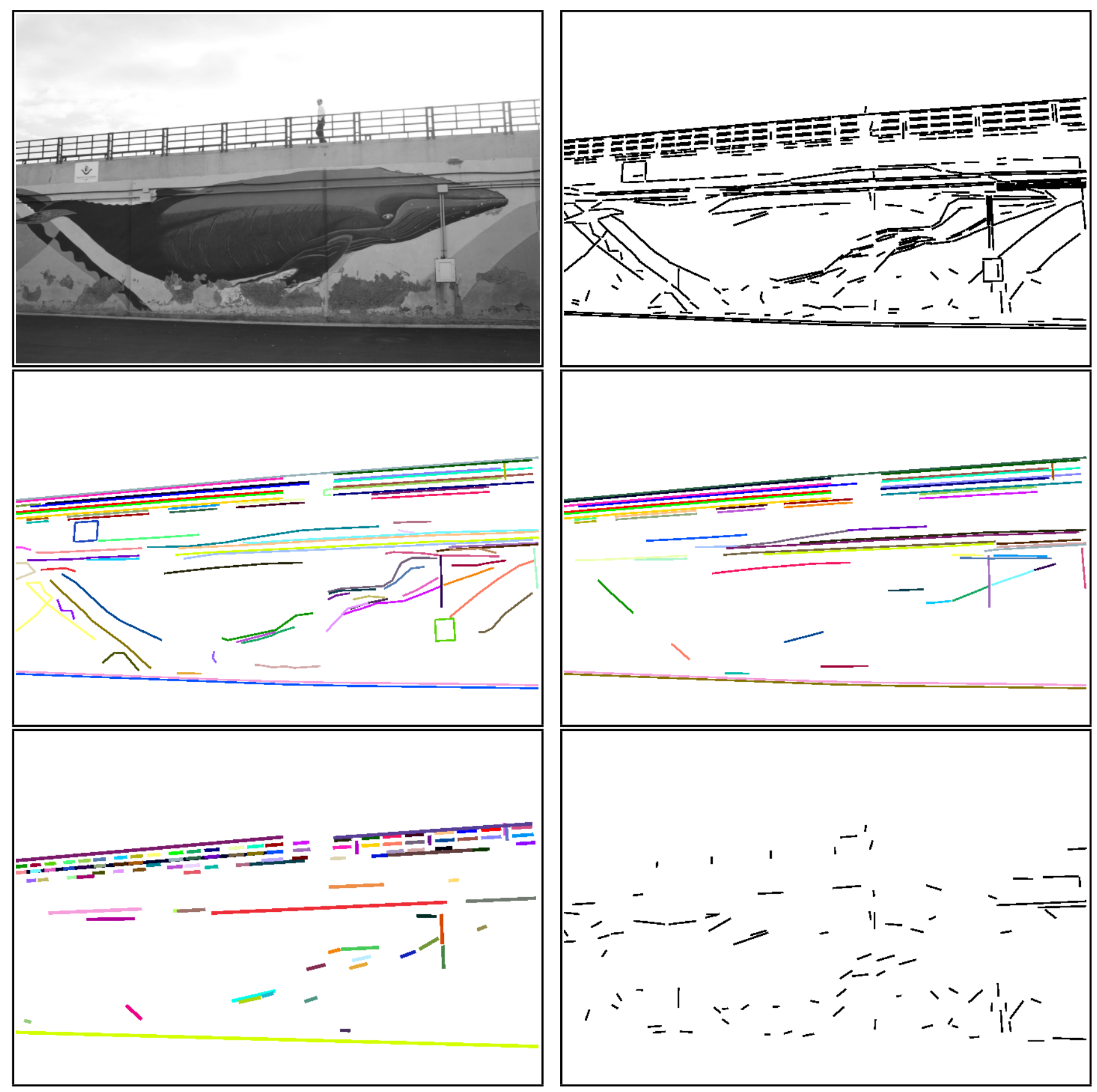

Figure 3: Gestalt detector performance over the Whale image - each color indicates one detected structure. From left to right and top to bottom: initial image, LSD line segments, all good continuations, non-local alignments, pairs of parallel line segments (bars), and finally all line segments that do not belong to any of the former structures. A line segment can belong simultaneously to several of these higher order partial Gestalts.

ments must be explained by T-junctions; bars and non-local alignments may be grouped again with the same good continuation and parallelism principles. These further steps are required to solve the figure-background problem by unsupervised algorithms.

We also notice that in several examples the found good continuations are not perceptually adequate. It is clear that convex good continuations should be given a preference over good continuations with oscillating direction, and that more specialized detectors detecting regular polygons or convex polygons seem a necessary refinement for the automatic analysis of human made structures. Furthermore our limitation of good continuation to 10 successive segments was due to computational 

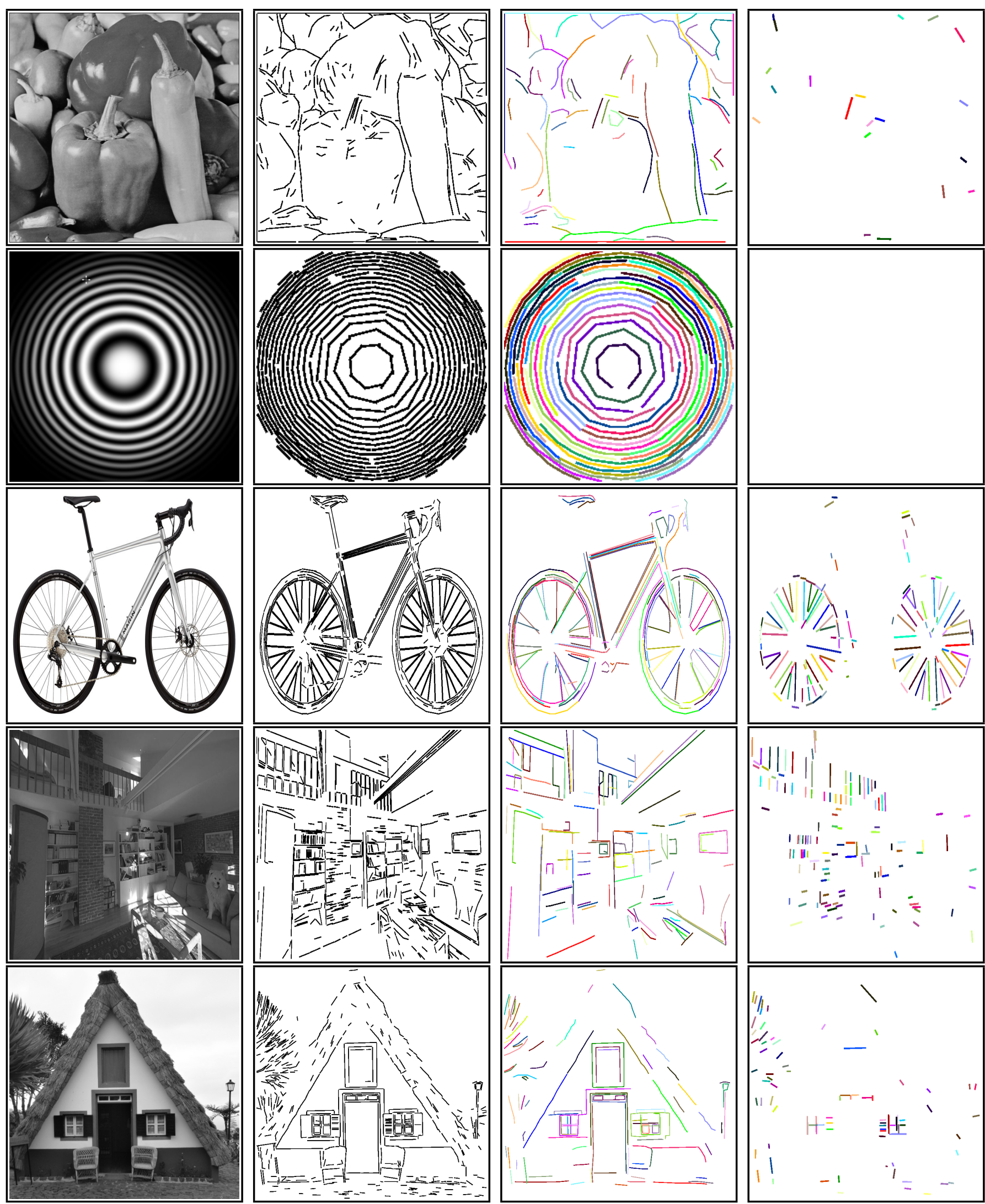

Figure 4: Good continuation and parallel line segment grouping - each color indicates one structure. From left to right, columns show original image, LSD line segments, good continuations and bars, respectively, for (from top to bottom) Peppers, Circles, Bicycle, Room and House images. 

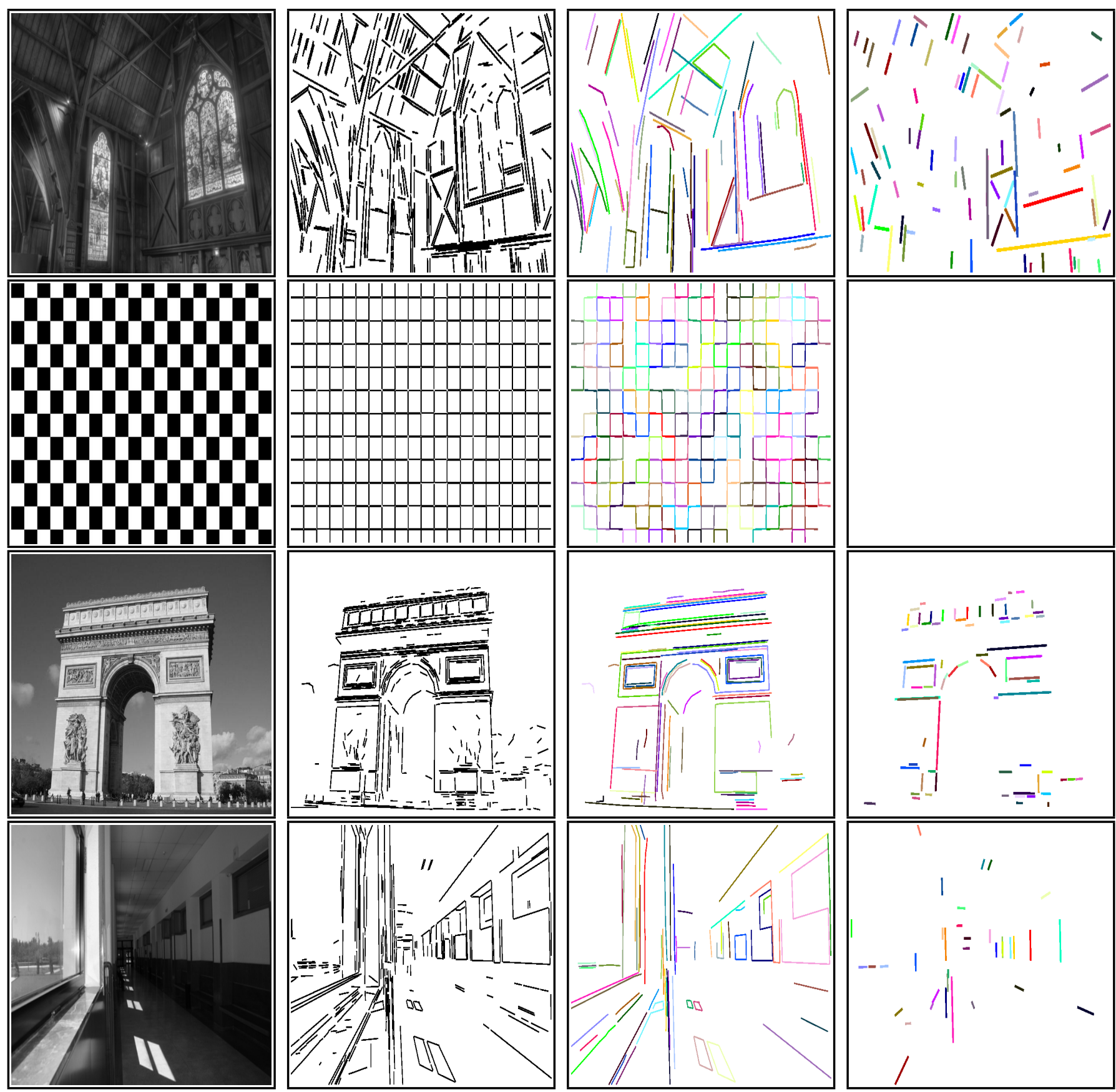

Figure 5: Good continuation and parallel line segment grouping - each color indicates one structure. From left to right, columns show original image, LSD line segments, good continuations and bars, respectively, for (from top to bottom) Church, Chess, Arc and Corridor images.

concerns only, and should be relaxed in future, more complete, implementations.

\section{Image Credits}

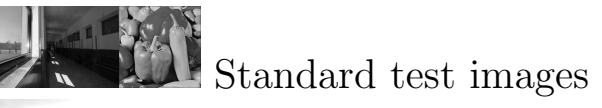

Jean-Michel Morel (CMLA, ENS-Cachan, France) 
(1).)

Pascal Getreuer (CMLA, ENS-Cachan, France)

Image by authors

15. photograph courtesy Philip Greenspun (http://philip.greenspun.com)

Dean S. Pemberton (dean@deanpemberton.com)

(.). 0

Free credit from http://www.marinbikes.com

[4. Saved from http://demo.ipol.im/demo/69/

Saved from http://demo.ipol.im/demo/145/

\section{References}

[1] C. Akinlar and C. Topal, Edlines: A real-time line segment detector with a false detection control, Pattern Recognition Letters, 32 (2011), pp. 1633-1642. https://doi.org/10.1016/j. patrec.2011.06.001.

[2] __, Edcircles: a real-time circle detector with a false detection control, Pattern Recognition, 46 (2013), pp. 725-740. https://doi.org/10.1016/j.patcog.2012.09.020.

[3] M. K. Albert and D. D. Hoffman, Genericity in spatial vision, in Geometric Representations of Perceptual Phenomena: Articles in Honor of Tarow Indow's 70th Birthday, D. Luce, K. Romney, D. Hoffman, and D’Zmura M., eds., Erlbaum, 1995, pp. 95-112. .

[4] N. Burrus, T. M. Bernard, and J. M. Jolion, Bottom-up and top-down object matching using asynchronous agents and a contrario principles, in Computer Vision Systems, Springer, 2008, pp. 343-352.

[5] _ Image segmentation by a contrario simulation, Pattern Recognition, 42 (2009), pp. 15201532. https://doi.org/10.1016/j.patcog.2009.01.003.

[6] F. CAO, Application of the Gestalt principles to the detection of good continuations and corners in image level lines, Computing and Visualization in Science, 7 (2004), pp. 3-13. https: //doi.org/10.1007/s00791-004-0123-6.

[7] J. Cardelino, V. Caselles, M. Bertalmío, and G. Randall, A contrario hierarchical image segmentation, in Proceedings of IEEE International Conference on Image Processing, IEEE, 2009, pp. 4041-4044. https ://doi.org/10.1109/ICIP. 2009.5413723.

[8] A. Desolneux, L. Moisan, And J. M. Morel, Meaningful alignments, International Journal of Computer Vision, 40 (2000), pp. 7-23. https://doi.org/10.1023/A:1026593302236.

[9] A. Desolneux, L. Moisan, and J. M. Morel, From Gestalt Theory to Image Analysis, Springer, 2008.

[10] D. Gerogiannis, C. Nikou, And A. Likas, Elimination of outliers from $2 D$ point sets using the Helmholtz principle., IEEE Signal Processing Letters, 22 (2015), pp. 1638-1642. https: //doi.org/10.1109/LSP.2015.2420714. 
[11] R. Grompone von Gioi, J. Jakubowicz, J. M. Morel, and G. Randall, LSD: A fast line segment detector with a false detection control, IEEE Transactions on Pattern Analysis and Machine Intelligence, 32 (2010), pp. 722-732. https://doi.org/10.1109/TPAMI.2008.300.

[12] _ LSD: a Line Segment Detector, Image Processing On Line, (2012). https://doi.org/ 10.5201/ipol.2012.gjmr-lsd.

[13] B. Grosjean and L. Moisan, A-contrario detectability of spots in textured backgrounds, Journal of Mathematical Imaging and Vision, 33 (2009), pp. 313-337. .

[14] G. Kanizsa, Organization in vision: Essays on Gestalt perception, Praeger New York:, 1979.

[15] J. Lezama, R. Grompone von Gioi, G. Randall, and J. M. Morel, Finding vanishing points via point alignments in image primal and dual domains, in IEEE Conference on Computer Vision and Pattern Recognition, 2014, pp. 509-515. .

[16] J. Lezama, J. M. Morel, G. Randall, and R. Grompone von Gioi, A contrario 2D point alignment detection, IEEE Transactions on Pattern Analysis and Machine Intelligence, 37 (2015), pp. 499-512. https://doi.org/10.1109/TPAMI.2014.2345389.

[17] D. Lowe, Perceptual Organization and Visual Recognition, Kluwer Academic Publishers, 1985.

[18] W. Metzger, Gesetze des Sehens, Verlag Waldemar Kramer, Frankfurt am Main, third ed., 1975 .

[19] P. Musé, F. Sur, F. Cao, Y. Gousseau, and J. M. Morel, An a contrario decision method for shape element recognition, International Journal of Computer Vision, 69 (2006), pp. 295-315. https://doi.org/10.1007/s11263-006-7546-0.

[20] V. Pătrăucean, P. Gurdjos, and R. Grompone von Gioi, A parameterless ellipse and line segment detector with enhanced ellipse fitting, in European Conference on Computer Vision, 2012. .

[21] J. Rabin, J. Delon, And Y. Gousseau, A statistical approach to the matching of local features, SIAM Journal on Imaging Sciences, 2 (2009), pp. 931-958. https://doi.org/10. $1137 / 090751359$.

[22] B. Rajaei, R. Grompone von Gioi, and J. M. Morel, From line segments to more organized Gestalts, IEEE Southwest Symposium on Image Analysis and Interpretation, (2016), pp. 137-140. https://doi.org/10.1109/SSIAI.2016.7459194.

[23] I. Rock, The Logic of Perception, The MIT Press, 1983. ISBN $=0262181096$.

[24] N. Sabater, A. Almansa, And J. M. Morel, Meaningful matches in stereovision, IEEE Transactions on Pattern Analysis and Machine Intelligence, 34 (2012), pp. 930-942. https: //doi.org/10.1109/TPAMI.2011.207.

[25] S. Sarkar And K. L. Boyer, Perceptual organization in computer vision: A review and a proposal for a classificatory structure, IEEE Transactions on Systems, Man and Cybernetics, 23 (1993), pp. 382-399. https://doi.org/10.1109/21.229452.

[26] J. Wagemans, J. H. Elder, M. Kubovy, S. E. Palmer, M. A. Peterson, M. Singh, AND R. VON DER HEYDT, A century of Gestalt psychology in visual perception: I. perceptual grouping and figureground organization., Psychological Bulletin, 138 (2012), pp. 1172-1217. http://dx.doi.org/10.1037/a0029333. 
[27] J. Wagemans, J. Feldman, S. Gepshtein, R. Kimchi, J. R. Pomerantz, P. A. VAN DER Helm, AND C. VAN LeEuWen, A century of Gestalt psychology in visual perception: II. conceptual and theoretical foundations., Psychological Bulletin, 138 (2012), pp. 1218-1252. http://dx.doi.org/10.1037/a0029334.

[28] M. Wertheimer, Untersuchungen zur Lehre von der Gestalt. II, Psychologische Forschung, 4 (1923), pp. 301-350. https://doi.org/10.1007/BF00410640.

[29] N. Widynski And M. Mignotte, A contrario edge detection with edgelets, in Proceedings of International Conference on Signal \& Image Processing Applications, 2011, pp. 421-426. https://doi.org/10.1109/ICSIPA.2011.6144087. 